[Aus der I. medizin. (Direktorial)-Abteilung (Prof. Dr. Deneke) des Allgem. Krankenhauses St. Georg, Hamburg.]

\title{
Erfahrungen über Hausinfektionen im großen allgemeinen Krankenhause.
}

Von

Dr. Artur Lippmann.

Trotz aller Errungenschaften im Erkenmen und Behandeln ansteckender Krankheiten, die die zweite Hälfte des 19. Jahrhunderts dem Krankenhausarzte gebracht haben, und trotz der reichen Mittel, die ihm in technischer und bavlicher Beziehung zur Bekämpfung dieser Krankheiten zur Verfügung gestellt wurden, blieb das Krankenhaus nicht verschont von immer wieder auftretenden mehr oder weniger zahlreichen Fällen von Ansteckungen unter seinen Pfleglingen. Man hat sich gewöhnt, diese Fälle (Hausinfektionen) als im gewissen Grade unvermeidlich anzusehen, und war im allgemeinen zufrieden, wenn ihre Zahl nicht so erheblich stieg, daß sie den eigentlichen Krankenhausbetrieb lahmlegte, sei es durch Personalmangel oder die Notwendigkeit störender Verlegungen oder Platzmangel bei gebotenen zahlreichen Isolierungen. Die traurigen, nur durch Hausinfektionen bedingten 'Todesfälle seien trotz ihrer sicher überall nicht geringen Zahl hier nur erwähnt, ebenso wie die viel häufigeren folgenschweren Fälle, in denen eine schon gesicherte Rekonvaleszenz durch eine Hausinfektion gestört und ein erneuter langer Krankenhausaufenthalt erforderlich wird.

Ein eigentliches Diskussionsthema bilden diese Hausinfektionen nicht, nur gelegentlich wird anläßlich des Neubaues einer Krankenhausanlage die Frage angeschnitten, ob sich nicht die Hausinfektionsgefahr beseitigen oder verringern ließe. Ś besprach neuerdings wieder Feer ${ }^{1}$ die Frage

I Die Verhiitung der Übertragung ansteckender Krankheiten in den Spitälern. Korrespondenzblatt f. Schw. Ärxte. 1916. Bd. XLIII. 
eingehend, vor allem auch unter Erörterung geschichtlicher Gesichtspunkte, aus denen hervorgehoben sei, daß zucrst in Frankreich, etwa um das Jahr 1889, eigentliche Isolierabteilungen eingerichtet wurden, und da $\beta$ damit erst die größte Ansteckungsgefahr, die vorher beim Zusammenlegen aller möglichen Infektionskrankheiten bestand, . etwas eingedämmt wurde. Isolierstationen machten immer weitere Fortschritte, indem bald nicht mehr die Gruppenisolierung genügte, sondern jeder einzelne Kranke im Einzelzimmer behandelt wurde. Demgenı̈̈ $B$ waren aber auch die Erfolge erheblich bessere, so daß die schon vorher erreichte, als günstig angesehene Zahl von 3 Prozen Hausinfektionen auf $0 \cdot 3$ Prozent eingeschränkt werden konnte. Jiese Erfahrungen beziehen sich nun allerdings nur auf reine Spezialabteilungen für Kinder. Allgemeine Krankenhauserfahrungen über Hausinfektionen sind in Deutschland in größerem Maße jedenfalls nicht regelmäßig veröffentlicht worden, so daß wir glauben, mit der von Deneke an unserem Krankenhause zuerst eingefülırten regelmäBigen Bearbeitung der Hausinfektionsfrage der allgemeinen Krankenhaushygiene einen Dienst erwiesen zu haben. Das Krankenhaus veröffentlicht nämlich seit 1910 jährlich in seinem Jahresbericht ${ }^{1}$ die genauen Zahlen und den Weg der Hausinfektion. Daneben wurden in anderen Arbeiten kritische Zusammenstellungen über diese Frage gegeben." Die folgende Zusammenstellung soll nın noch einma] mit Gegenüberstellen von Kriegs- und Friedensjahren dis Material der letzten 8 Jahre geben und kritisch beleuchten.

Als Unterlagen dienen Meldebögen, die bei uns über jeden Fall von Hausinfektionen geführt werden. Sie enthalten auf einem doppelseitigen großen Bogen außer den üblichen Daten Aufnahmetag, Saal- und Bettnummer, Angaben über bisherige Krankheiten, Bettlägerigkeit, Beschäftigung, dann den Tag der Erkrankung an der Hausinfektion mit dem wahrscheinlichen Zeitpunkt der Ansteckung; ferner die Namen sämtlicher an der Pflege und Behandlung Betejligten mit Zeitangaben, falls Wechsel stattfand. Die Frage des Zustandekommens der Infektion (Auffihrung der im selben Raume vorgekommenen anderen Infektionsfälle, Berührungspunkte, Möglichkeit der Einschleppung durch Urlaub oder Besuch, weitere Infektionsquellen (wie Wasser, Milch), ergriffene MaBregeln (wie Tag der Verlegung, dann Desinfektion, Behandlung der im selben Raume Zurückbleibenden), ferner Verlauf der Infektion (Heilung, Gesundheitsstörungen, Tod usw.) und schließlich Aufzählung der weiteren Infektionen, die auf den Fall zurückzuführen waren.

Die Uberwachung der Hausinfektion geschieht durch den Sekundäraræt der inneren Abteilung, die genaue Kontrolle durch den Direktor.

Das Krankenhaus selbst ist im Pavillonsystem erbaut. Die Pavillons

1 Jahrbiicher der Hamburger Staatskrankenanstalt. $1910 \mathrm{u}$. ff.

${ }^{2}$ Zeitschrift für Krankenanstalten. 1914. 13d. XXI. 
enthalten meistens in zwei Stockwerken $80 \mathrm{Kranke}$ in vier Sälen mit einzelnen Nebenzimmern. Daneben bestehen einige größere Gebäude (Hantabteilung, Frauenkrankheiten), die in Häusern mit Korridorsystem je etwa $200 \mathrm{Kranke}$ unterbringen. Die eigentliche Infektionsabteilung liegt räumlich getrennt von der übrigen Abteilung am Ende der Anstalt. Das Pflegepersonal dieser Abteilung schläft und ißt getrennt von dem übrigen Personal. Diese Abteilung hat einen Scharlach- und einen Diphtheriepavillon und ein Doppelhaus mit zwei Eingängen für Keuchhustenkranke und auf der anderen Seite eine Abteilung für Masern und Mischinfektionen.

Das Krankenhaus hatte in den fraglichen Jahren durchschnittlich 1900 Betten, davon für Kinder und Säuglinge rund 250. Die Säuglingsbetten machten je nach den Jahren verschieden von den Kinderbetten die Hälfte bis ein Drittel aus. Seit 1913 liegt die eigentliche Säuglingsabteilung räumlich getrennt von der Kinderabteilung, während sio vorher nicht geschieden war. Die eigentliche Säuglingsabteilung hat 30 Betten, die anderen Säuglingsbetten sind verteilt auf die geburtshilfliche, chirurgische, Kinder- und auf die einzelnen Infektionsabteilungen. Durch den Krieg wurde die Bettenzusammensetzung insofern erheblich geändert, als einerseits durch die Hinzunahme einer Schule die Bettenzahl erheblich vermehrt wurde und andererseits anfänglich durch Reduktion der Betten für Zivilisten die Bettenzahl für Soldaten in erster Linie gesteigert wurde, so daß eine Zeitlang die Zivilaufnahmen zurücktraten. Diese Fragen spielen aber für die Betrachtung dèr Hausinfektionen in einem allgemeinen Krankenhause keine ausschlaggebende Rolle, denn wie wir gleich sehen werden, spielt das kindliche Alter auch im allgemeinen Krankenhause allein die ausschlaggebende Rolle, so daß eine 1917 notwendig gewordene Erhöhung der Kinderbetten von 251 auf 295 exheblich wichtiger ist.

Tab. I zeigt nach den Ergebnissen des Jahres 1917 die Altersverteilung der Kranken, die von einer Hausinfektion heimgesucht wurden. Man sieht, daß rund ein Fünftel der Fälle das Säuglingsalter betreffen und daß neun Zehntel aller Hausinfektionen Kinder bis zum 10. Lebensjạhre ergriffen haben, eine Tatsache, die nicht weiter verwunderlich ist, wenn man sieht, daß die Hausinfektionen im wesentlichen in Kinderkrankheiten bestehen. Typhus, der sonst noch in Betracht käme, spielt numerisch eine verschwindend kleine Rolle; Ruhr, Pocken und andere Krankheiten kommen überhaupt kaum in Betracht. Erwachsene werden eigentlich nur von Diphtherie und in stärkerem Maße von Scharlach bedroht, während für die Säuglinge neben Diphtheric und Másern in erster Linie Wasserpocken und Keuchhusten in Frage kommen; Scharlach ist, wie aueh alte Erfahrungen lehren, für das frühe Säuglingsalter ohne große Bedeutung. Als Wesentlichstes ergibt also die Tabelle, daß auch im allgemeinen Krankenhause der wesentliche Anteil der Hausinfektionen auf die Infektion der Kinder entfällt, von denen vor allem das Spielalter gefährdet ist. 
Tabelle $\mathrm{I}$.

Von 100 Hausinfektionen einer Krankheitsart entfallen auf die verschiedenen Altersstufen' folgende Anteilzahlen:

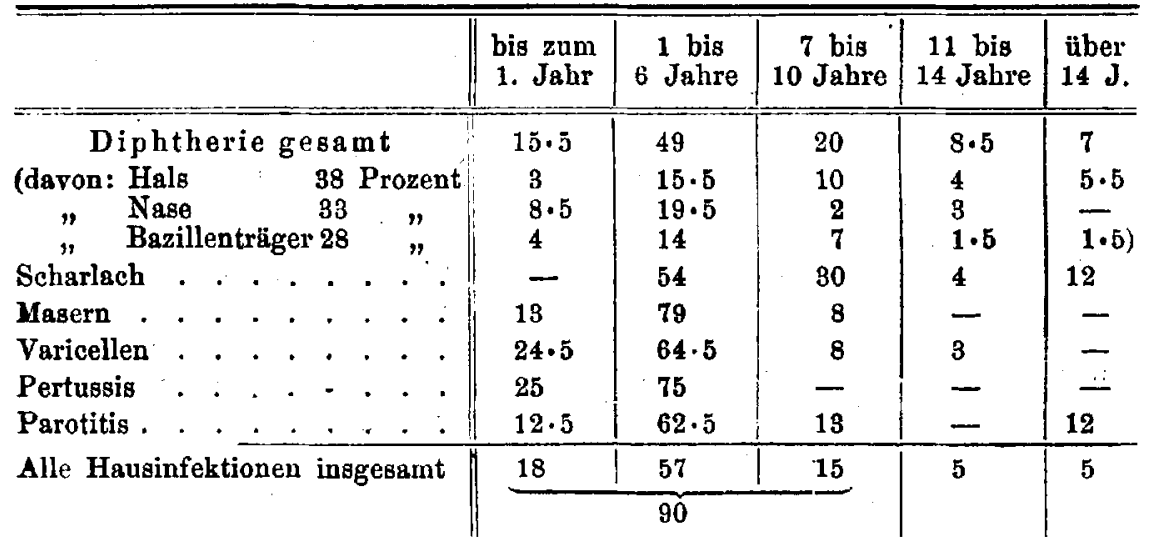

Die Tab. II zeigt nun, wieviel Fälle von Hausinfektionen sich in jedem Jahre ereignet haben. Hierbei ergibt sich nun, daß das Verhalltnis der einzelnen wichtigeren Gruppen, wie Diphtherie, Scharlach, Masern, untereinander gar nicht sehr wesentlich schwankt, während Wasserpocken und Keuchhusten in einzelnen Jahren erheblich verstärkt auftraten. Diphtherie nimmt immer bei weitem die erste Stelle ein, es folgen Scharlach und Masern. Die im Kriege eingetretene Änderung tußert sich im wesentlichen darin, daß die Diphtheriezabl, die im Frieden erfreulich abgesunken war; wieder anstieg, und daß vor allem Wasserpocken im letzten Jahre sehr zunahmen.

Die Gesamtzahl der Hausinfektionen ist im Kriege erheblich gestiegen und das bei einer stark zurückgegangenen Aufnahmezahl, so daB der Eindruck zuerst noch bedenklicher erscheint (auf 1000 Aufnahmen vor dem Kriege 7, im Kriege 12 Hausinfektionen), jedoch ist diese einfache Berechnung irreführend. Bei dem andersartigen Krankenmaterial, das im Kriege zu einem Drittel aus Soldaten bestand, $\mathrm{mu} \dot{\mathrm{B}}$ die Statistik auch von anderer Seite betrachtet werden. Die tägliche Belegung ging keineswegs zurück, die Behandlungsdauer des einzelnen Kranken (Soldaten vor allem) wurde erheblich höher, so dab die Summe der Behandlungstage die der Friedenszeit übertraf. Die tägliche Belegungs-

1 Prozentsatz für Säuglinge, für Kinder bis zu 10 Jahren

$\begin{array}{llr}\text { Krankenbetten } & 4.5 & 9 \\ \text { Aufnahmezahl } & 0.6 & 19 \\ \text { Behandlungstage } & 0.6 & 14\end{array}$


T'abelle II.

\begin{tabular}{|c|c|c|c|c|c|c|c|}
\hline & 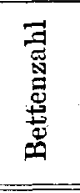 & 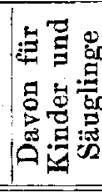 & 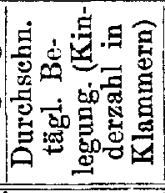 & 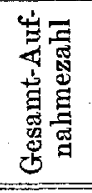 & 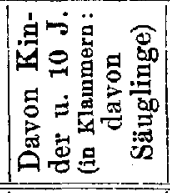 & 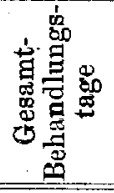 & 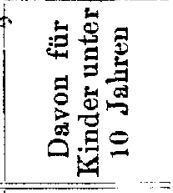 \\
\hline 19 & 1574 & 218 & $1349(172)$ & 19884 & $2524(118)$ & 493123 & 62382 \\
\hline 1 & 1 & 222 & 1426 (187) & 22177 & $2888(153)$ & 52 & 661 \\
\hline 1912 & 1779 & 264 & $1533 \quad(200)$ & 23478 & 3062 (214) & 5611 & 72493 \\
\hline 19 & 177 & 254 & $1628(217)$ & 23657 & $3201(356)$ & 593 & 77577 \\
\hline 19 & 1 & 253 & $1596(206)$ & 22655 & $3042(251)$ & 58 & 74542 \\
\hline 1915 & 22 & 254 & $1737(211)$ & 19321 & $3134(170)$ & 633729 & 76327 \\
\hline & & 25 & $1623(214)$ & 178 & $3126(108)$ & 593 & \\
\hline & & 29 & $1605(240)$ & 17494 & 3499 (11) & 586049 & 86815 \\
\hline Durchschnitt & 1903 & $251^{1}$ & $1587(206)$ & 20800 & $3059(185)$ & 570530 & $74415(3436)$ \\
\hline
\end{tabular}

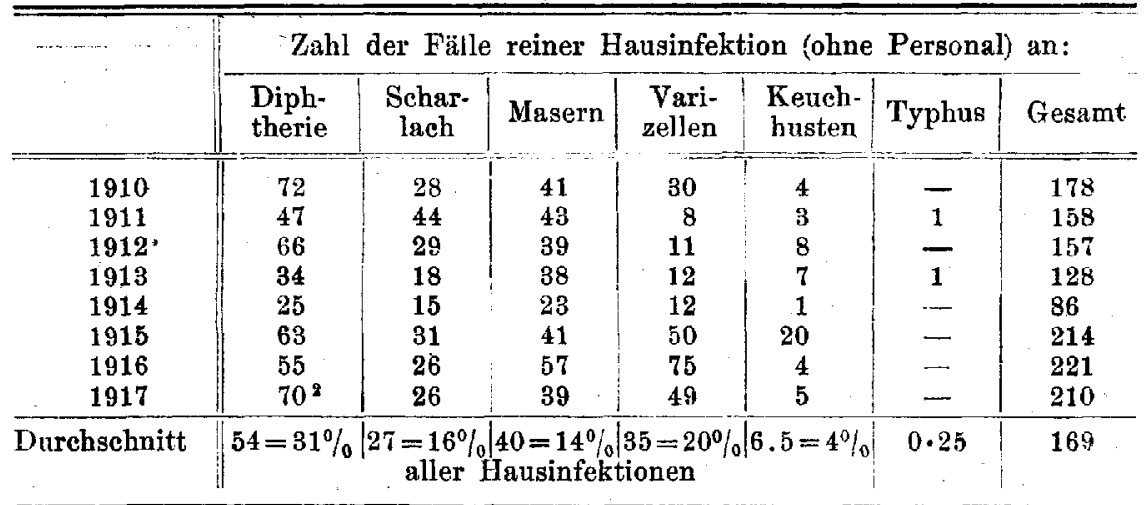

\begin{tabular}{|c|c|c|c|c|c|c|c|c|}
\hline & \multicolumn{8}{|c|}{ Es entfallen von allen Hausinfektionen auf: } \\
\hline & 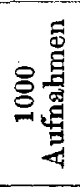 & 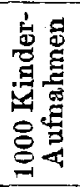 & 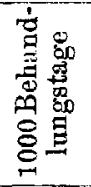 & 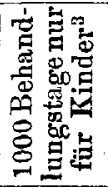 & 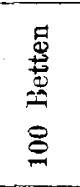 & 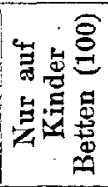 & 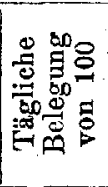 & 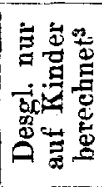 \\
\hline 1910 & 8.4 & 63 & 0.36 & 2.5 & $11 \cdot 3$ & 78 & 13 & 93 \\
\hline 1911 & 7. & 49 & 0.33 & $2 \cdot 0$ & 9.8 & 64 & 11 & 76 \\
\hline 1912 & $6{ }^{\circ}$ & 46 & 0.28 & 1.9 & 8.8 & 53 & 10 & 70 \\
\hline 1913 & 5.4 & 36 & 0.22 & 1.5 & 7.2 & $4 \check{b}$ & 8 & 53 \\
\hline 1914 & $3 \cdot 8$ & 25 & 0.15 & 1.0 & 4.4 & 30 & 5 & 37 . \\
\hline 1915 & 11.8 & 62 & 0.34 & 2.5 & 9.7 & 76 & 12 & 91 \\
\hline 1916 & $12 \cdot 3$ & 63 & 0.37 & $2 \cdot 6$ & $10 \cdot 2$ & 79 & 14 & 93 \\
\hline 1917 & $12 \cdot 0$ & 54 & 0.36 & $2 \cdot 2$ & $9 \cdot 7$ & 64 & 13 & 79 \\
\hline Durchschnitt & $8 \cdot 4$ & 50 & 0.29 & $2 \cdot 0$ & $8 \cdot 3$ & 60 & 11 & 74 \\
\hline
\end{tabular}

1 Davon durchschnittlich 100 für Säuglinge.

2 Auberdem 27 Bazillenträger.

a Bei der Berechnung der Anteilzahlen der Hausinfektionen der Kinder werden die 10 Proz. in Abzug gebracht, die Personen über 10 Jahre betrafen. 
zahl war somit auch höher als im Frieden, so daß sich die erhöhte Zahl der Hausinfektionen trotz der verringerten Aufnahme auf mehr Kranke verteilte. Die Berechnung für eine tägliche Belegungszahl von 100 zeigt nun auch, daß die Aussicht, sich eine Hausinfektion zu erwerben, im Kriege nicht so erheblich gestiegen ist, wie es die erste Uberlegung scheinen lieB. Sie ist aber doch einwandsfrei vorhanden. Wie die Tab. I lehrt, spielt nun die Zahl der erwachsenen. Kranken für die Zahl der Hausinfektionen keine große Rolle (10 Prozent). Die Zahlen müssen deshallb für die behandelten Kinder berechnet werden, wobei sich dann allerdings sehr hohe Zahlen für Hausinfektionen ergeben. Eine getrennte Statistik nur für Kinder wird bei uns hinsichtlich der Aufnahmezahlen und Behandlungstage nicht geführt; Kincer wercen unter. Männer und Frauen, je nach dem Geschlecht, werrechnet. Es ergab sich deshalb für die Betrachtung unserer Zahlen die Notwendigkeit, die den Kindern zukommenden Zahlen aus dem Verhältnis der gesamten Veıpflegungstage für Kinder zu ilrer durchsehnittlichen Behandlungsdauer zu berechnen, die damit doch noch hinreichend genau werden. Es fällt bei dieser Berechnung an der an und für sich schon schr hohen Zahl der Hausinfektionen für Kinder auf, daß im Kriege, abgesehen von der absoluten Steigerung der Infektionszahlen für Kinder, die ja anch durch die steigende Belegungszahl mit Kindern zum Teil erklärt wird, anch ein deutliches Ansteigen der prozentualen Ziffern stattfindet, so steigt die Prozentzahl der Ansteckungen auf 100 Kinderbetten von 59 vor dem Kriege auf 73 im Kriege und die entsprechende auf die tägliche Bolcgungszahl berechnete von 73 auf 88.

Die Zahl der Hausinfektionen, die auf 1000 Aufnahmen fallen und die bei uns durchschnittlich 8.4 beträgt, läßt an und für sich keine weiteren Schlüsse zu und erlaubt keinen Vergleich mit den Zahlen anderer Krankenhäuser, solange nicht die durchselunittliche Behandlungsdauer und Belegung des Hauses und die Zusammensetzung (Erwachsene und Kinder) des Krankenmaterials bekannt ist. Die Zahl 8 Promille als solche wïrde immerhin ein recht gutes Ergebnis darstellen. (Paris z. B. 30 Promille), wemn nicht unsere. relativ geringe Kinderzahl unter den Kranken als verschlechtemdes Moment hinzutritt. Für uns bedeutet doch diese Zahl, daß im Durehschnitt bei einem Krankenbestand vón 1000 täglich 0.3 Erkrankungen vorkommen oder bei einem Krankenbestand von 3330 täglich 1 Hausinfektionsfall. Nur für die Kinder gerechnet, heißt dieses aber schon auf 100 behandelte Kinder täglich 0.2 Fälle oder auf 500 Kinder täglich 1 Fall. Der Unterschied zwischen Frieden und Krieg tritt hier bei einer getrennten Berechnung auld noch hervor. 
So würde sich jeden Tag 1 Infektionsfall ereignen bei einem Krankenbestand von

1910 bis 19133700 insgesamt oder bei 493 Kindern

$$
1915,, 19172770 \quad, \quad, \quad, 410 \quad \text {, }
$$

Die Folgen dieser zahlreichen Hausinfektionen sind eingangs erörtert worden. Neben der schweren Sehädigung des Betriebes der Anstalt spielen vor allem die Todesfälle eine traurige Rolle, die unbedingt auf die Hausinfektion bezogen werden müssen. Die Tab. III ergibt für derartige Todesfälle jährlich wenigstens 15 Fälle, die alle Kinder betrafen, so da. auf 100 Kinderbetten 6 Todesfälle im Jahre eintraten. Betroffen werden meistens Kinder mit Masern und Diphtherie, bei denen im Krankenhaus eine zweite Infektionskrankheit hinzukommt, oder auch Rachitiker. Daneben spielt die wichtigste Rolle die Zahl der Kinder, die durch eine Hausinfektion aus guter Rekonvaleszenz gerissen oder auf ein langes Krankenbett geworfen werden. Die schwere Schädigung ihrer Gesundheit wiegt bei der großen Zahl derartiger Fälle fast ebenso schwer wie die Todesfälle.

Tabelle III.

Gestorben an Hausinfektion:

\begin{tabular}{l||c|c|c|c}
\hline \multicolumn{1}{c|}{ Durchschnittlich an: } & Dipht. & Scharl. & Masern & Pertuss. \\
\hline \hline Jährliche Fälle 15.3, davon & $4 \cdot 6$ & 2.5 & $\mathbf{5 . 8}$ & 0.6 \\
(Niedrig/höchst) $7: 27$ & $1: 8$ & $1: 8$ & $3: 7$ & $1: 2$
\end{tabular}

Auf Krankenzahl $0.7 \%$

Auf Kinderzahl allein berechnet $5.0 \%$

Auf Bettenzahl 0.8 Proz., nur auf Kinderbetten bereehnet 6.1 Proz.

Auf tägliche Belegung 1 Proz., auf tägliche Belegung nur für Kinder berechnet 7.0 Proz.

Die Zahl der jährlich an Hausinfektion erkrankenden Mitglieder des Personals zeigt die Tab. IV, wobei aber betont werden muß, daB hier unter dem Personal das gesamte in der Anstalt wohnende verstanden ist, das eigentliche Pflegepersonal macht aber weniger als die Hälfte aus. Da es natürlich erheblich mehr als das Arbeiter- und Reinmachepersonal einer Infektion ausgesetzt ist, so ist der .Prozentsatz der Erkrankungen unter dem eigentlichen Pflegepersonal erheblich höher als der Doppelte des angegebenen. An erster Stelle der Erkrankungen steht hier wieder die Diphtherie, Scharlach tritt sehr zurüsk, Masern und Typhus spielen kaum eine Rolle. Insgesamt haben wir eine jährliche Erkrankungsziffer von 2.5 Prozent. Die meisten Fälle eignen sich bei der Pflege Infektions- 
Tabelle IV.

Hausinfektionen unter dem gesamten im Hause wohnenden Personalı

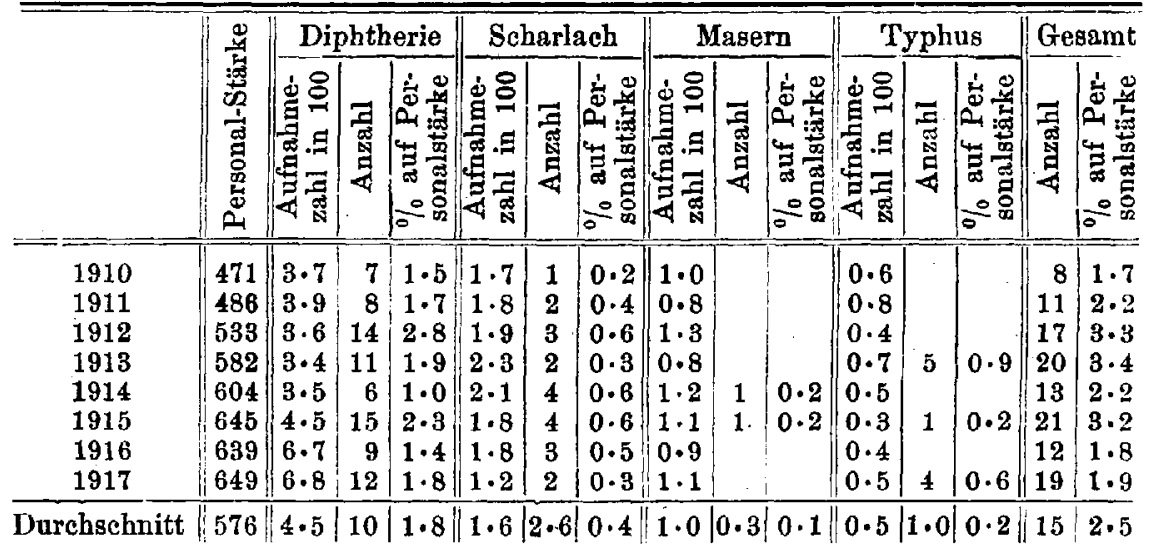

kranker, doch ist immerhin bei einer ganzen Reihe von Fällen (bei Typhus die Hälfte, bei Diphtherie auch zahlreich) keine unmittelbare Ansteckungsquelle im Krankenhause herauszufinden, so daß eine Ansteckung außerhalb des Hauses für viele Fälle in Betracht kommt. Auf die große Gefahr, die infiziertes Pflegepersonal für Kranke darstellt, wird noch eingegangen werden.

Es erhebt sich nun die wichtigste Frage, nämlich: Von welchen Faktoren ist die Zahl der Hausinfektionen abhängig und wie können wir sie einschränken? Zuerst könnte man an ein Verhältnis zwischen der Zahl der im Hause aufgenommenen Infektionskranken und der im Hause erfolgten Ansteckungen denken. Dies ist nun nach unserem Material nicht der Fall. Auf Tab. IV sind die jährlichen Aufnahmezahlen der wichtigsten Infektionskrankheiten mit angegeben. Vergleicht man diese mit der Zahl der Hausinfektionen auf Tab. II, so crribt sich hier keine Abhängigkeit der Zahlen voneinander. Nur für die Diphtherie könnte man vielleicht ein gewisses Parallelgehen dieser Zahlen herausfinden, doch erlaubt gerade Diphtherie durchaus nicht den Schluß, daß durch die steigende Aufnahme an Diphtheriekranken die Hausinfektionen an Diphtherie steigen müssen, sondern es spielen hier andere, noch zu erörternde Verhältnisse (Verbreitung von Bazillenträgern) die ausschlag-

1 Das eigentliche Pflegepersonal macht hiervon etwa 50 Proz. aus, die übrigen 50 Proz. bestehen nus Bedienungs-, Reinigungs- und Arbeiterpersonal. Die Prozentzahl für das eigentliche Pflegepersonal allein ist somit über das Doppelte höher. 
gebende Rolle. Auch eine Abhängigkeit der Zahh der Hausinfektionen von der Erkrankungszahl des Personals läßt sich nicht finden.

Nach unseren jedes Jahr wieder neugewonnenen Erfahrungen ist dagegen der wesentlichste Grund zum Zustandekommen der Hausinfektionen die Aufnahme und das Verlegen schon infizierter, im Inkubationsstadium befindlicher Kranker auf eine Abteilung für Nichtansteckende. Kinder, die keinerlei Zeichen einer schon stattgehabten Ansteckung aufweisen, werden z. B. mit einem frischen Knochenbruch auf eine chirurgisclse Kinderabteilung verlegt, wo nun einige Tage nachher bei ihnen z. B. Masern ausbrechen, oder Kinder mit einem als harmlos imponierenden Husten entpuppen sich auf einer gewöhnlichen Kinderabteilung dann allerdings meist zu spät als Keuchhustenkinder. Die Zahl dieser infiziert aufgenommenen und auf falsche Abteilungen Verlegten zeigt die Tab. V.

Tabelle V.

Infiziert aufgenommen, auf falsche Abteilungen gelegt wurden:

\begin{tabular}{|c|c|c|c|c|c|c|c|c|c|c|c|}
\hline & \multirow[b]{2}{*}{ 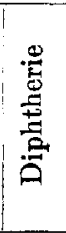 } & \multirow[b]{2}{*}{ 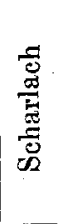 } & \multirow[b]{2}{*}{  } & \multirow[b]{2}{*}{ 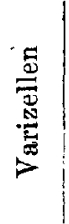 } & \multirow[b]{2}{*}{ 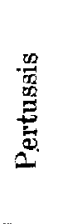 } & \multirow[b]{2}{*}{$\frac{0}{3}$} & \multirow[b]{2}{*}{$\begin{array}{l}\text { Infektionen } \\
\text { Gesamt }\end{array}$} & \multicolumn{2}{|c|}{$=\%$} & \multicolumn{2}{|c|}{$\begin{array}{l}\% \text { auf } \\
\text { Betten }\end{array}$} \\
\hline & & & & & & & & 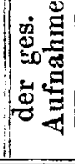 & 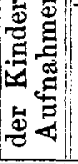 & $\cong$ & 罾 \\
\hline 1910 & 2 & 2 & 7 & 3 & - & - & 14 & 0.7 & 5.5 & 0.9 & $6 \cdot 4$ \\
\hline 1911 & 6 & 5 & 6 & . & - & - & 18 & 0.8 & 6.2 & 1.1 & $s \cdot 1$ \\
\hline 1912 & 7 & 5 & 5 & 4 & 2 & . & 29 & $1 \cdot 2$ & 0.4 & 1.7 & 11.0 \\
\hline 1913 & 12 & 4 & 7 & $\overline{5}$ & 2 & . & 31 & 1.8 & $9.7 \mid$ & 1.9 & 12.2 \\
\hline 1914 & 8 & 3 & 3 & 1 & 5 & 1 & 23 & 1.0 & $|.7 \cdot 5|$ & 1.4 & $9 \cdot 1$ \\
\hline 1915 & 1 & 6 & 3 & 3 & 1 & 3 & 18 & 0.9 & 5.7 & 1.0 & $7 \cdot 1$ \\
\hline 1616 & 4 & 3 & 6 & 1 & 1 & 1 & 16 & 0.9 & $5 \cdot 1$ & 1.0 & $6 \cdot 4$ \\
\hline 1917 & 2 & 4 & 7 & 3 & - & - & 19 & $1 \cdot 0$ & $5 \cdot 4$ & $1 \cdot 2$ & $6 \cdot 4$ \\
\hline Durchschnitt & $5 \cdot 3$ & 4 & $5 \cdot 5$ & $|2 \cdot 5|$ & $1 \cdot 4$ & 0.6 & 21 & $1 \cdot 0$ & $6 \cdot 8$ & $1 \cdot 3$ & $8 \cdot 3$ \\
\hline $\begin{array}{c}=\% \text { der ges. } \\
\text { Aufnahmezahl } \\
\text { an der betreff. } \\
\text { Krankheit }\end{array}$ & 1 & $2 \cdot 5$ & $5 \cdot 5$ & 22 & $1 \cdot 6$ & $1 \cdot 2$ & $\begin{array}{l}\text { ungefiihr } 2.3 \\
\text { aller aufge- } \\
\text { nommenen In- } \\
\text { fektionskrank- } \\
\text { heiten }\end{array}$ & & & & \\
\hline
\end{tabular}

Die durchschnittliche Zahl dieser Aufnahmen scheint nieht sehr hoch, handelt es sich doch für jede Krankheitsart jäbrlich nur um wenige Fälle, die z. B. für Diphtherie nur 1 Prozent der gesamten Diphtherieaufnahmen, für Scharlach $2 \cdot 5$, für Masern $5 \cdot 5$, für Varizellen bei allerdings wenigen Fällen aber schon über 20 Prozent ausmachen. Doch spielt diese Zahl sofort eine ganz andere Rolle, wenn man sich klarma,cht, 
welche Folgen diese einzeln aufgenommenen Fälle für eine Kinderabteilung haben. So können wir Jahr für Jahr nachweisen, daß weit über die Hälfte unserer Masern-Hausinfektionen auf eine Ansteckung durch infiziert Aufgenommene zurückzuführen sind, für Varizellen bleibt diese Ansteckungsart sicher die Regel. Für Scharlach tritt sie erheblich zurück, wälırend sie für Keuchhusten in einzelnen Jahren wieder die ausschlaggebende Rolle spielte, vor allem weǹn es sich um Säuglingsabteilungen gehandelt hat. Für Diphtherie liegen die Verhältnisse erheblich schwieriger. Hier spielt neben der Ansteckung durch nicht erkannte Diphtherieerkrankung die Ansteckung dureh die erheblich häufigeren Bazillenträger unter den Aufnahmen die größte Rolle. Ansteckung von einer nicht erkannten Rachendiphtherie ausgehend, ist selten, denn bei nur einigermaßen sorgfältiger Beobachtung auf einer Krankenabteilung wird die Rachendiphtherie rechtzeitig orkannt werden. Aber ein großer Teil der Diphtherie-Hausinfektionen erfolgt von Kindern (meist Säuglingen) mit Nasendiphtllerie. Harmloser Schnupfen im jugendlichen Alter entpuppt sich nur zu häufig als Nasendiphtherie; ein blutiger, ätzencer Ausfluß braucht dabei gar nicht vorhanden zu sein. Von dem einfachen Schnupfen zu einer fast trockenen und gänzlich gesund aussehenden Nase, die aber reichlich Diphtheriebazillen beherbergt, finden sich viele tbergänge, und diese "gesunden" Nasen mit reichlich Diphtheriebazillen sind auch sehr häufig unter den Aufnahmen vertreten. Diese Fälle muB man schon zu den Bazillenträgern rechnen, zu der Gruppe von Fällen, bei denen Aufnahmen ohne jede Krankheitserscheinung meist im Rachensekret Diphtheriebazillen haben. Diese Bazillenträger sind je nach der Ausbreitung der in der Stadt herrschenden Epidemie mohr oder weniger zahlreich. In großen Untersuchungsreihen haben wir $1910 \mathrm{z}$. B. feststellen können, daß rund 10 Prozent sämtlicher an anderen Krankheiten zur Aufnahme kommenden Krankenhausinsassen Diphtheriebazillenträger waren. ${ }^{1}$ Natürlich bilden derartige Kranke eine große Gefahr für ihre Ungebung, vor allem auf Kinderabteilungen. Diese Gefahr ist nun noch erheblich höher, wenn unter dem Pflegepersonal ebenfalls Diphtheriebazillenträger sind, denn bei der engen Berührung zwischén Pflegerin und krankem Kind z. B. ist beinahe das Nichtübertragen als Ausnahme anzusehen. In der eben zitierten Arbeit wurde auch gezeigt, daß als Ursache einer langdauernden Diphtherieendemie im Krankenhause Bazillenträger unter dem Pflegepersonal anzusehen waren. Ein langer und weit verzweigter Weg der Diphtherieausbreitung im Hause ließ sich unter dem Personal nachweisen und immer

- Beobachtungen an Diphtheriebazillenträgern. Diese Zeitschrift. Bd. LXVII. 
wieder endeten diese Wege bei neuen Ansteckungsfällen unter den Kranken, womit erwiesen war, daß jedenfalls für die Diphtherieverbreitung im Krankenhause Bazillenträger unter Kranken und Personal eine ausschlaggebende Rolle spielen; durch einfache fortlaufende Untersuchung des Personals und Herausziehen der Bazillenträger aus der Kinderpflege lic $B$ sich die Endemie ohne weitere Maßregeln beendigen. Wir sahen somit, da $B$ infiziert Aufgenommene und Bazillenträger, die als Verbreiter des Ansteckungsstoffes ihnen gleichstehen, die Hauptrolle bei der Entstehung von Hausinfektionen spielten, die für Frieden und Krieg wohl dieselbe Bedeutung gehabt hat, es sei denn, daB im Kriege die Untersuchung auf Diphtheriebazillenträger nicht so häufig und gründlich stattfinden konnte.

Ein zweiter wichtiger Punkt ist der, daß unsere Infektionskranken nicht immer sicher genug isoliert wurden; sei es, daß auf der eigentlichen Infektionsabteilung mehrere Gruppen von Infektionskrankheiten in einen schädlichen Kontakt miteinander kamen oder da $B$ auf der Abteilung für Nichtansteckende verdächtige Kranke in Einzelzimmern isoliert wurden, aber nicht mit den nötig strengen Mabregeln und dem gewünschten Erfolg. Unsere Infektionsabteilung hat zwar in eigenen Häusern für Masern und Diphtherie die Möglichkeit, diese ganzen Gruppen gut abzusondern, da aber sowohl im Scharlach- wie im Diphtheriehause nur zwei bzw. nur ein kleines Isolierzimmer zur Verfügung steht, ergeben sich bei Misch-. infektionen oder schon beim ersten Auftreten einer Hausinfektion große Schwierigkeiten. Die Folge ist, daß wir sowohl im Scharlach- wie im Diphtheriehause verhältnismäßig viel Hausinfektionen haben, die natürlich zu einem Teil damit zu begründen sind, daß das dort behandelte Krarkenmaterial, eben Kinder, ganz besonders zu Hausinfektionen disponiert ist; und andererseits gerade der Scharlach auch nach den Erfahrungen anderer Krankenhäuser den günstigsten Boden zu einer Infektion abgibt. Diphtherie, Varizellen und auch vereinzelt Masern sind häufige*Gäste auf der Scharlachabteilung. Im letzten Berichtsjahre hatten wir in diesem Pavillon die hohè Zahl von 10 Prozent Hausinfektionen bei sämtlichen aufgenommenen Scharlachkranken; Diphtheriekranke, die besonders für Scharlach und Masern disponiert sind, erkrankten dagegen nur in 3 Prozent der Fälle. Unsere Masernabteilung liegt unter demselben Dache wie die Keuchhustenabteilung, die Abteilungen haben vollständig getrennte Eingänge und Wirtschaftsräume, gruppieren sich aber um einen gemeinsamen Hof, der dem Personal doch manche Berührungspunkte, gestattet. Auf beiden Abteilungen sind an einem kleinen Korridor mehrere Einzelzimmer vorhanden, in denen je nach der Krankenzahl die Masern-bzw. Keuchhustenkranken 
oder Mischinfektionen oder auch andere Krankheiten (wie Varizellen) behandelt werden. Die Wirtschaftsräume jeder dieser Abteilungen sind aber für den ganzen Hausteil gemeinsam, so daß sich hier ebenso wie auf dem kurzen gemeinsamen Gange das Personal dauernd trifft. Die Folge dieses engen Zusammenlebens und Aneinanderreibens sind verhältnismäBig häufige Hausinfektionen der Kinder, die durch Verschleppung von Ansteckungsstoffen mit Geschirr, Wäsche und Händen, in manchen Fällen wohl auch durch eine reine Luftübertragung zu erklären sind.

Klarer wird dieser Punkt noch, wenn man sich den Gang einer Hausinfektion auf einer Abteilung für Nichtansteckende vergegenwärtigt. Hier bilden sich in der Regel um einen infiziert aufgenommenen, nicht erkannten Infektionsfall mehr oder weniger begrenzte Herde, für deren Ausdehnung die Art der thbertragung des Krankheitsstoffes ausschlaggebend ist. Bei Übertragung durch direkten Kontakt werden die Bettnachbarn ergriffen, bei indirektem Kontakt (Wäsche, nicht desinfizierte Hände der Pflegerinnen) erkrankte häufig eine Gruppe von Kranken, die eine gemeinsame Pflegeperson hat, bei reiner Ubertragung durch die Luft sahen wir wiederum, da $B$ alle ergriffenen Vorsichtsmaßregeln nichts fruchteten und da $B$ ein ganzer Saal angesteckt wurde. Für die Hauptgruppen der Hausinfektionen läßt sich bei jahrelanger Beobachtung im. Krankenhause eine Meinung über ihre gewöhnliche Verbreitungsart bilden, die hier nochma]s kurz zusammengefa Bt sei.

Die Diphtherieansteckung wird, soweit nicht, wie oben erörtert, Bazillenträger unter dem Pflegepersonal die Kranken durch Tröpfchenverstäubung aus Mund und Nase anstecken, häufig auch durch Bazillenträger unter den Kranken verbreitet. Diese gesunden Individuen laufen im Saale herum, Kinder spielen miteinander usw., und durch diesen innigen Verkehr werden die Keime von Mund zu Mund verbreitet. Laufende Nasen (mit ,+, Bazillenbefund) geben dem Personal der Kinderabteilung nur zu leicht Gelegenheit, die Hand beim Ausschnupfen der Kinder zu beschmutzen, um dann bei im guten Glauben unterlassener Desinfektion eine Utbertragung auf den nächsten Kranken hervorzurufen. Die Diphtheriebazillen werden nach unserer Erfahrung in der Regel von Mensch zu Mensch direkt verbreitet, seltener ist die indirekte Verbreitung durch mit Sekret beschmutzte Gegenstände und Pflegerhände. Luftübertragung mit Staub möchten wir ausschließen, denn nach Verlegung des Kranken hört jede Infektion auf. Einen Schutz bietet nur neben der absolut sicheren Isolierung jedes Diphtheriefalles einschließliclı der Bazillenträger ein dauerndes Kontrollieren des Pflegepersonals durch häufiges Abimpfen auf Diphtheriebazillen. Außerdem ist jede Neuaufnahme mit 
nur irgendwie verdächtiger Fumilienanamnese und jede laufende Nase ausnahmslos abzuimpfen.

Für den Scharlach kommt ebenfalls in erster Linie direkter Kontakt als Ansteckungsquelle in Betracht, wobei Nasen- und vor allem Rachensekret den Ansteckungsstoff vermittelt. Ubertragung durch Schuppen haben wir nicht einwandfrei feststellen können, jedenfalls bleibt bei jeden Falle von Scharlachübertragung durch ein schuppendes Kind der Rachen immer noch am verdächtigsten. Wir hatten den Eindruck, daß das Scharlachvirus sehr widerstandsfähig ist, so daß auch noch nach Entfernung des Kranken längere Zeit eine Ansteckung in dem von ihm vorher belegten Raume stattfinden kann. Besonders gefährdet sind stets Kinder mit frischen Wunden (Operation, Verbrennung), von denen jährlich einige bei uns erkrankten. Hier muß allerdings auch die Vermutung ausgesprochen werden, daß die gewöhnliche Art der Hausübẹrtragung nicht dafür ausschlaggebend ist, sondern daB durch die Wunde das Virus leichter und unter Umgehung der Abwehrmaßregeln in den Körper eindringen kann. Die Scharlachbekämpfung im Krankenhause ist nicht schwierig, sofortige Isolierung des ersten Falles (Fieberanstieg mit Angina) führt meistens zum Erfolg.

Bci Masern sicht man fast regelmäßig um den auf der Abteilung für Nichtansteckende schon infiziert aufgenommenen, aber erst nach einigen Tagen zum Ausbruch kommenden Masernfall Gruppen von anderen Fällen auftreten, und zwar ist diese Ansteckung schion besiegelt, wenn man die Frühdiagnose der Masern mit dem ersten Auftreten der Koplikschen Flecke stellt. Die tbertragung findet cinwandfrei durch Anhusten statt, so daß die entfernter liegenden Kinder, wenn nicht unglückseligerweise durch Pflegerinnenhände Auswurf verschleppt wird, ganz im Anfang noch vor der Infektion zu retten sind. Das Virus selbst hält sich nur kurze Zeit in der Luft, denn nach Entfernung des Kranken ist auch jede Gefahr beseitigt. Nötig ist aber, selbst die anscheinend gesunden Nachbarn des ersten Masernfalles von vornherein auf 12 Tage zu isolieren, damit diese Kinder bei der, wie sicher anzunehmen ist, folgenden Infektion nicht wieder im späten Inkubationsstadium Herde für neue Fälle abgeben.

Die Varizellenübertragung erfolgt wohl sicher durch Verstäubung des Materials der eingetrockneten Bläschen, wahrscheinlich auch durch Rachensekret. Jedenfalls spielt neben Ubertragung durch das Personal die Luftübertragung die größte Rolle, denn hei allen Vorsichtsmaßregeln gelingt es nicht, bei Auftreten von Varizellenfällen einen Saal freizuhalten, sondern bis in die äußersten Ecksen und nicht gut abgeschlossenen Neben- 
zimmer wird die Infektion übertragen, so daß meist alle Vorsichtsmabregeln zu spät kommen.

Pertussis scheint nach allen Erfahrungen am ansteckendsten nur im katarrhalischen Stadium zu sein, ehe man durch das Auftreten typischer Hustenanfälle die Keuchhustendiagnose stellen kamm. Die Ubertragung findet durch Anhusten und natürlich anch durch die Pflegerinnenhände statt. Wir hatten den Eindruck, daß der Keuchhusten im. Stadium convulsivum wenig oder gar nicht mehr ansteckt.

Parotitis e pidem. wird anseheinend auch nur direkt vom Kranken übertragen. Durch möglichst frülle Isolierung konnten wir Weiterverbreitung stets verhüten.

Typhusübertragung auf Patienten hatten wir, obwohl Typhuskranke bei uns auf allgemeinen inneren Stationen in Einzelzimmern behandelt werden, so gut wie gar nicht. Ubertragung auf das Personal crfolgt nach alter allgemein angenommener Erklärung meist durch die Exkremente, sicher auch vereinzelt durch Anhusten. Der Schutz des Pflegepersonals ist damit gegeben und besteht in einwandfreier Händedesinfektion und Schutzimpfung.

Die Rolle, die das Pflegepersonal bei der Entstehung der Hausinfektionen spielt, ist nach obigem sehr bedeutend, sei es im aktiven Sinne, indem es unmittelbar Ansteckungsiiberträger ist, oder im passiven, indem es drohende Ansteckung durch Nichterkennen der Gefahr nicht verhütet.

Das geringe Ansteigen der Zahl der Hausinfektionen im Kriege möchten wir deshalb auch zum Teil damit erklären, daß das Personal nach Abgabe eines alten erfahrenen Stammes zum Kriegsschauplatz in dem Erkennen und Bekämpfen der üblichen Infektionskrankheiten nicht so hochwertig war wie friiher, wozu dann auch noch anfangs eine Uberbürdung mit Arbeit trat. Der sonst so oft als Sündenbock bezeichnete Seifenmangel spielt sicher keine ausschlaggebende Rolle, da Desinfektionsmittel stets reichlich vorhanden waren.

Neben den oben gegebenon speziellen Verhütungsmaßregeln gegen einzelne Krankheiten hat nun noch das große allgemeine Krankenhaus allgemeine Schutzmaßregeln zu ergreifen. Vor allem kommt, da, wie aus Tab. I ersichtlich, wie verschieden die einzelnen Lebensalter beteiligt sind, eine Trennung der Erwachsenen von den Kindern und der Kinder wiederum nach Lebensalter in Betracht. Säuglinge müssen stets von älteren Kindern getrennt untergebracht werden, so daß die allgemeine Säuglingsabteilung sämtliche nichtansteckenden Säuglinge aufnimmt, auch die chirurgisch Kranken, um sie damit von den 
anderen Kindern abzusondern. Das.Spielalter ist für sich unterzubringen, was schon deshalb erforderlich ist, um die Schulkinder, deren Anamnese in bezug auf Ansteckung immer am dunkelsten ist, von den am meisten gefährdeten Kindern, eben den vom 2. bis 6. Lebensjahre, nach Möglichkeit fernzuhalten. Die dritte Abteilung umfaßt endlich die eigentlichen Schulkinder, wobei noch einmal die Wichtigkeit einer guten Anamnese, die sich auf alle Krankheiten im Hause und in der Schulklasse bezieht, hervorgehoben sei.

Bei der Aufnahme der Kranken, vor allem aber der Kinder, ist natürlich mit gröbter Sorgfalt zu verfahren. Das ideale Verfahren, alle neu aufzunehmenden Kinder so lange zu isolieren, bis der Ausbruch einer Infektionskrankheit unmöglich ist, ist im allgemeinen Krankenhausbetriebe bei dem regen Wechsel des Bestandes aus Platzmangel unmöglich. Man muß sich darauf beschränken, alle nur irgendwie körperlich oder: durch die Anamnese Verdächtigen zu isolieren; dazu ist eine große Reihe Isolierzimmer, möglichst mit nur einem Bett, nötig. Mischinfektionen sollen nicht in die Häuser für Scharlach und Diphtherie verlegt werden, sondern ebenso wie alle unklaren Infektionsfälle in einem eigenen Hause vereinigt werden, für das wir (vor allem nach den Erfahrungen Feers) ein einfaches Haus mit vielen Einzelzimmern im Korridorsystem, in dem gemeinsame Gebrauchsgegenstände in einem Desinfektionsraume entkeimt werden, für das günstigste halten, vorausgesetzt, daß gut ausgebildetes Pflegepersonal zur Verfügung steht. Krankenverlegungen innerhalb der Anstalt dürfen unter keinen Umständen, wenn in der Abteilung Hausinfektionen vorkamen, früher stattfinden, als bis die Inkubationszeit für mögliche neue Ansteckungen abgelaufen ist, was nur zu leicht vergessen wird.

Auf das Erkennen des ,ersten“ Falles oder der ersten symptome einer ausbrechenden Infektionskrankheit ist dureh gewissenhafte Temperaturmessung und durch besondere Schulung des Personals der Hauptwert zu legen.

Als bestes Erziehungsmittel und die beste Überwachung für Ärzte und Pflegepersonal ist schließlich die gewissenhafte Meldung und Verfolgung der Hausinfektionsfälle anzusehen, für die wir als Muster die Art der Meldungen, wie sie mit den Zählblättern betreffend Hausinfektionen im Krarkenhaus St. Georg eingeführt sind, ansehen: Durch das genaue Durchforschen und Offenlegen der Hausinfektionen wird im Kumpfe gegen diesen Feind am meisten erreicht werden, sowohl durch die Erziehung als auch durch die Furcht, allzu häufig in diesen Meldungen als beteiligte Pflegeperson gènannt zi werden. 